\title{
Dietary glycaemic load and intakes of carbohydrates, fats and proteins in 1040 hospitalised adult Chinese subjects
}

\author{
$\mathrm{Li} \mathrm{Lv}^{1 *}$, Yushi $\mathrm{YaO}^{2}$ and Lei Wang ${ }^{1}$ \\ ${ }^{1}$ Department of Clinical Nutrition, General Hospital of Chinese People's Armed Police Forces, Beijing 100039, \\ People's Republic of China \\ ${ }^{2}$ Department of Biotherapy, General Hospital of the Beijing Military Commanding Region, Beijing 100700, \\ People's Republic of China \\ (Received 12 October 2010 - Revised 16 February 2011 - Accepted 16 February 2011 - First published online 11 April 2011)
}

\section{Abstract}

Metabolic diseases in China have been on the rise during recent decades. To investigate dietary glycaemic load (GL) and its correlation with the prevalence of metabolic diseases in hospitalised Chinese adults, we analysed the total energy and macronutrient intakes in 1040 patients. Dietary GL was calculated based upon glycaemic index (GI), carbohydrate content and daily intake of individual foods. Dietary patterns at different dietary GL levels and the distribution of dietary GL were analysed in patients from the internal medical departments $v$. those from surgical departments. Height and weight were measured using a standardised protocol. The present study results demonstrated that in hospitalised adults, $95.5 \%$ of dietary GL came from cereals. Average GL was higher in male patients than in female patients, and was higher in patients from surgical departments than in patients from medical departments. Patients with lower dietary GL consumed inadequate carbohydrates and excessive fats, and therefore had a higher prevalence of overweight and obesity. Dietary GL analysis may be helpful to nutrition assessment and rational diet intervention in hospitalised Chinese patients.

\section{Key words: Glycaemic load: Nutrition assessment: Carbohydrate: Dietary patterns: Hospitalised adult Chinese}

Dietary carbohydrates are closely associated with the health of the human body. In 1997, Walter Willett and colleagues proposed the concept of dietary glycaemic load (GL), which was used to reflect the quality and quantity of dietary carbohydrates $^{(1)}$. Though controversial, the relationship between dietary GL and disease has attracted much attention from the nutrition community and has been used in dietary assessment worldwide ${ }^{(2-4)}$.

The conventional Chinese dietary pattern is characterised by a high energy ratio of carbohydrates and relatively low energy ratios of protein and fat. However, metabolic diseases among the Chinese have been continuously increasing, presumably due to altered dietary patterns associated with improving economic circumstances during the past decades. As misunderstandings in the crucial role of carbohydrates are quite prevalent among Chinese individuals, dietary assessments and guidelines are urgent in China. Dietary GL that reflects dietary carbohydrates directly and the overall situation of the daily diet might be an optimal indicator that represents dietary patterns and the risks of metabolic diseases, though needing to be warranted through investigation. In the present study, we analysed dietary GL in 1040 hospitalised Chinese adults, seeking to verify the application of dietary GL in dietary assessment among the Chinese population.

\section{Experimental methods}

\section{Subjects}

Participants were consecutively selected in the present study. A total of 1040 adult Chinese hospitalised in the General Hospital of Chinese People's Armed Police Forces from January 2009 through to November 2009 were selected. Patients with severe impairment of cardiac, hepatic or renal functions and those not able to eat orally were rejected. Fasting patients or those on a strictly limited diet (for example, partial parenteral nutrition) at any time on days $1-3$ of hospitalisation were also excluded from the study. The participation rate was $88.7 \%$ and 132 patients were excluded. The average age of all the 1040 patients (624 male and 416 female, 516 from surgical departments and 524 from internal medical departments) was 49 (range 19-65) years. The male:female ratios in the surgical department and internal medical department were $1 \cdot 36: 1(297 / 219)$ and $1 \cdot 65: 1(327 / 197)$, respectively. The present study was conducted according to the guidelines

Abbreviations: GI, glycaemic index; GL, glycaemic load.

* Corresponding author: Dr Li Lv, fax +8610 88276688, email crystalleilei@yahoo.com.cn 
laid down in the Declaration of Helsinki and all procedures involving patients were approved by the Ethics Committee of the General Hospital of Chinese People's Armed Police Forces. The application of personal medical data in the present study was approved by all patients enrolled. Glycaemic index (GI) data were from the Food Composition Table of China $(2002)^{(5)}$ and international GI tables ${ }^{(6)}$.

\section{Data analysis}

Dietary GL/d was calculated according to dietary GI and the daily consumption of various foods. Daily dietary $G L=\Sigma$ (food GI $\times$ daily quantity of carbohydrates obtained from that food); sex and department distributions of dietary GL were analysed. Daily dietary GL of food $\mathrm{X}=(\mathrm{GL}$ of food $\mathrm{X} /$ daily dietary GL) $\times 100 \%$. Carbohydrate intake was positively proportional to energy intake, and so was dietary GL. In order to compare carbohydrate intake with the same level of total energy intake, daily energy intakes in all patients in the present study were adjusted to $10460 \mathrm{~kJ} / \mathrm{d}$. GL in the Abstract, Results and Discussion sections represents GL corrected for $10460 \mathrm{~kJ}$ (GL/10 $460 \mathrm{~kJ})$.

\section{Baseline measurements}

Diets of patients were recorded on days $1-3$ of hospitalisation and average nutrient intake was calculated. During their hospitalisation, participants could order foods according to their own diet habits from a nutrition canteen in our hospital. Therefore, all foods (including fruits and snacks, weighed as precisely as $0 \cdot 1 \mathrm{~g}$ ) for all the enrolled patients were provided by the hospital. In order to guarantee the accuracy of food weighing, residual foods that were not eaten by patients were recovered and quantified. Thus, dietary patterns of these patients during hospitalisation could reflect their daily dietary patterns to a maximum extent. Body mass and height for each patient were determined by nurses in charge according to one uniform criterion. Weight was measured without shoes and in light clothes to the nearest $0.1 \mathrm{~kg}$ by using a beam balance scale. Height was measured to the nearest $0 \cdot 1 \mathrm{~cm}$ by using a stadiometer.

\section{Classification of overweight and obesity}

In the present study, we used the most common BMI classification for Chinese adults. According to the Chinese standards $^{(7,8)}$, BMI of $<24 \mathrm{~kg} / \mathrm{m}^{2}$ is classified as normal weight, BMI of $24-27.9 \mathrm{~kg} / \mathrm{m}^{2}$ is classified as overweight and BMI of $\geq 28 \mathrm{~kg} / \mathrm{m}^{2}$ is classified as obese. Diagnosis of the metabolic syndrome was in accordance with the diagnostic criteria published in 2004 by the Diabetes Branch of the Chinese Medical Association ${ }^{(9)}$.

\section{Statistical analysis}

Data are presented as mean values and standard deviations. A $\chi^{2}$ test was used to compare the prevalence of overweight or obesity between groups. The statistical package SPSS for Windows (version 15.0; SPSS Inc., Chicago, IL, USA) was used for statistical analysis. $P<0.05$ was considered to be statistically significant.

\section{Results}

\section{Dietary glycaemic load in hospitalised adult Chinese}

General information of all the 1040 patients is shown in Table 1 . And, as can be seen in Table 2, mean daily dietary GL (256.7 in all participants) was higher in male patients (277.9) than in females $(231.3 ; P<0.05)$, and was higher in patients from surgical departments (278.6) than those from internal medical departments $(222 \cdot 0 ; P<0.01)$.

\section{Food origin of dietary glycaemic loads}

In the patients that we reviewed, $47 \cdot 27 \%$ of daily dietary GL came from rice, $40.73 \%$ from wheat, $3.98 \%$ from miscellaneous grain crops, $1.83 \%$ from potatoes, $1.75 \%$ from cakes and pastry, $1.43 \%$ from fried wheat, $1.42 \%$ from fruits, $0.67 \%$ from sugar-containing drinks, $0.37 \%$ from dairy foods, $0.27 \%$

Table 1. Characteristics of hospitalised adult Chinese participants (Mean values and standard deviations)

\begin{tabular}{|c|c|c|c|c|c|c|}
\hline & \multicolumn{2}{|c|}{ Total $(n$ 1040) } & \multicolumn{2}{|c|}{ Medical ( $n$ 524) } & \multicolumn{2}{|c|}{ Surgical ( $n 516)$} \\
\hline & Mean & SD & Mean & SD & Mean & SD \\
\hline \multicolumn{7}{|l|}{ Age (years) } \\
\hline All & $49 \cdot 0$ & $17 \cdot 3$ & 52.4 & 19.9 & 45.5 & 22.9 \\
\hline Male & 47.6 & $16 \cdot 8$ & $50 \cdot 8$ & $16 \cdot 9$ & $44 \cdot 1$ & $19 \cdot 6$ \\
\hline Female & $50 \cdot 4$ & $15 \cdot 7$ & $55 \cdot 0$ & $15 \cdot 3$ & 47.4 & $21 \cdot 3$ \\
\hline Weight (kg) & 66.9 & 8.7 & $65 \cdot 8$ & $7 \cdot 2$ & $68 \cdot 0$ & 7.5 \\
\hline BMI $\left(\mathrm{kg} / \mathrm{m}^{2}\right)$ & $23 \cdot 6$ & 5.5 & $23 \cdot 8$ & 4.9 & $23 \cdot 3$ & $5 \cdot 2$ \\
\hline Waist circumference $(\mathrm{cm})$ & 79.2 & $9 \cdot 1$ & 83.4 & 8.5 & 74.9 & $8 \cdot 3$ \\
\hline HbAlc (\%) & $5 \cdot 3$ & 1.2 & $5 \cdot 7$ & 1.3 & 4.9 & $1 \cdot 1$ \\
\hline TAG $(\mathrm{mmol} / \mathrm{l})$ & $1 \cdot 28$ & 0.63 & 1.35 & 0.42 & $1 \cdot 2$ & 0.36 \\
\hline Total cholesterol $(\mathrm{mmol} / \mathrm{l})$ & 4.83 & 0.94 & 4.91 & 1.02 & 4.72 & 0.98 \\
\hline LDL-cholesterol (mmol/l) & $2 \cdot 48$ & 0.66 & $2 \cdot 62$ & 0.77 & $2 \cdot 34$ & 0.92 \\
\hline HDL-cholesterol (mmol/l) & 1.23 & 0.51 & 1.29 & 0.43 & $1 \cdot 17$ & 0.46 \\
\hline LDL:HDL cholesterol ratio & $3 \cdot 16$ & $1 \cdot 1$ & 3.25 & 1.2 & 3.06 & 1.3 \\
\hline
\end{tabular}


Table 2. Dietary glycaemic loads in hospitalised Chinese adults (Mean values and standard deviations)

\begin{tabular}{|c|c|c|c|c|c|c|}
\hline & \multicolumn{2}{|c|}{ Total } & \multicolumn{2}{|c|}{ Medical } & \multicolumn{2}{|c|}{ Surgical } \\
\hline & Mean & SD & Mean & SD & Mean & SD \\
\hline Total & $256 \cdot 7$ & 79.2 & $222 \cdot 0$ & 71.5 & $278.6 \dagger$ & $82 \cdot 6$ \\
\hline Male & $277 \cdot 9^{*}$ & $87 \cdot 1$ & $245 \cdot 8$ & $77 \cdot 1$ & 300.5 & 97.5 \\
\hline Female & 231.3 & 71.2 & $199 \cdot 1$ & 68.4 & 259.3 & $81 \cdot 3$ \\
\hline
\end{tabular}

* Mean value was significantly different from that for all female participants $(P<0.05)$.

$\dagger$ Mean value was significantly different from that for the participants in the medical departments $(P<0.01)$.

from beans, $0 \cdot 1 \%$ from candies, $0 \cdot 06 \%$ from vegetables and $0.03 \%$ from nuts (data not shown). About $95.5 \%$ of dietary GL came from cereals and tubers (rice, wheat, potatoes, miscellaneous grain crops and fried wheat).

Dietary patterns in patients with various glycaemic load levels

Daily energy intakes in all subjects were adjusted to $10460 \mathrm{~kJ} / \mathrm{d}$, and macronutrient intakes among patients at various dietary GL levels are presented in Table 3. In both male and female patients with lower dietary GL levels, a lower dietary energy ratio of carbohydrates and a higher energy ratio of fats were observed. In patients whose dietary GL was lower than the 25th percentile (except for females in surgical departments), the average daily intake of cereals was less than $300 \mathrm{~g}$ (energy ratio of carbohydrates $<50 \%$ ), and that of fats was over $95 \mathrm{~g}$ (energy ratio of fats $>35 \%$ ). Patients from internal medical departments whose dietary GL was lower than the 50th percentile ate less than $300 \mathrm{~g}$ of cereals (energy ratio of carbohydrates $<55 \%$ ) and over $90 \mathrm{~g}$ of fats (energy ratio of fats $>35 \%$ ). Male patients in surgical departments whose dietary GL was lower than the 25 th percentile ate less than $300 \mathrm{~g}$ of cereals (energy ratio of carbohydrates $<50 \%$ ) and over $90 \mathrm{~g}$ of fats (energy ratio of fats $>30 \%$ ).

\section{Prevalence of overweight, obesity and the metabolic} syndrome, and mean BMI in hospitalised Chinese adults with various dietary glycaemic load levels

Analysis of the prevalence of overweight, obesity and the metabolic syndrome, and mean BMI in patients with various dietary GL levels showed that patients from internal medical departments whose dietary GL was either lower than the 5th percentile or higher than the 95th percentile had a higher prevalence of overweight, obesity and the metabolic syndrome $(P<0.05)$; patients in surgical departments whose dietary GL was lower than the 5 th percentile had a higher prevalence of overweight, obesity and the metabolic syndrome $(P<0.05)$. Both male and female patients whose dietary GL was lower than the 5 th percentile or higher than the 95th percentile had a higher prevalence of overweight $(P<0.05)$. In male patients with dietary GL levels lower than the 5th percentile, higher prevalences of obesity and the metabolic syndrome $(P<0.05)$ were observed. In female patients with dietary GL levels higher than the 95th percentile, higher prevalences of overweight and the metabolic syndrome $(P<0.05)$ were observed; however, there was a comparable prevalence of obesity $(P>0 \cdot 05)$ (see Table 4$)$.

\section{Discussion}

As an indispensable component in comprehensive therapy, nutrition treatment can greatly improve physical conditions of hospitalised patients. However, clinical nutrition intervention will not be optimised unless nutritional conditions of patients are fully understood. In the present study, we sought to investigate dietary GL in hospitalised adult Chinese in order to provide basic information for a more standard, rational and balanced therapeutic diet programme.

GI describes the blood glucose response after the consumption of a carbohydrate-containing test food relative to a carbohydrate-containing reference food, typically glucose or white bread. GI was originally designed as a guide on food selection for individuals with diabetes, in whom foods with low GI were recommended. Compared with GI, GL takes into account both the GI and the quantity of a given food, as the amount of food consumption is a major determinant of postprandial hyperglycaemia. A rational diet depends on an optimal energy composition of carbohydrates, fats and proteins. A low-GL diet has been recommended based on study results that dietary GL is associated with CHD, myocardial infarction, type 2 diabetes and cancer ${ }^{(10-14)}$. On the other hand, the GI of animal foods such as meat and animal oil, which is much lower than that of cereals, hardly has any contribution to dietary GL. However, it is an obvious violation of a rational and balanced diet if low dietary GL is followed by the means of excessive animal foods and inadequate carbohydrates. Therefore, dietary GL evaluation is closely related to its detailed food origin. Carbohydrates are the major energy source in the Chinese diet and provide about $50 \%$ of daily energy intake on average. Dietary GL is a reflection of categories and amounts of carbohydrates in daily foods: daily dietary $\mathrm{GL}=\Sigma$ (food GI $\times$ daily amount of carbohydrates from that food), making up for the shortfall of GI that only reflects carbohydrates in one single food. Moreover, it provides an alternative method for epidemiological study on the relationship between the quality and quantity of dietary carbohydrates and physical condition. We observed in the present study that cereals were the dominant source of dietary GL in hospitalised adult Chinese patients, which accounted for about $95.5 \%$ of total daily dietary GL. Thus, in accordance with the results from previous studies, dietary GL mainly reflects daily cereal intakes in hospitalised adult Chinese.

Studies on dietary GL depend on balanced energy intake. Dietary GL adjusted for total energy reflects carbohydrate intake with the same amount of energy intake. In the present study, dietary GL per $10460 \mathrm{~kJ}$ of energy intake was calculated. Daily energy intakes in all patients were adjusted to $10460 \mathrm{~kJ} / \mathrm{d}$, making the study results more convincing. Patients with higher dietary GL ate higher amount of cereals and lower amount of fats, with a higher energy ratio of carbohydrates and a lower energy ratio of fats. Therefore, 
Table 3. Dietary patterns at various levels (percentiles; P) of glycaemic load (GL)/10460 kJ for all participants and for males and females separately

\begin{tabular}{|c|c|c|c|c|c|c|c|c|c|c|c|c|c|c|c|}
\hline & \multicolumn{3}{|c|}{ P5 } & \multicolumn{3}{|c|}{ P25 } & \multicolumn{3}{|c|}{ P50 } & \multicolumn{3}{|c|}{ P75 } & \multicolumn{3}{|c|}{ P95 } \\
\hline & Total & Males & Females & Total & Males & Females & Total & Males & Females & Total & Males & Females & Total & Males & Females \\
\hline \multicolumn{16}{|l|}{ Total } \\
\hline Cereal intake $(\mathrm{g} / \mathrm{d})$ & 216 & 195 & 234 & 298 & 287 & 314 & 345 & 329 & 370 & 427 & 405 & 435 & 510 & 495 & 537 \\
\hline Fat intake $(\mathrm{g} / \mathrm{d})$ & $125 \cdot 6$ & $129 \cdot 2$ & $115 \cdot 7$ & $95 \cdot 7$ & 97.0 & $92 \cdot 1$ & $76 \cdot 3$ & 77.9 & 72.5 & $61 \cdot 2$ & $64 \cdot 0$ & 59.9 & 41.6 & $43 \cdot 1$ & 38.9 \\
\hline \multicolumn{16}{|c|}{ Energy contribution (\%) } \\
\hline Carbohydrates & 34.1 & $32 \cdot 8$ & $37 \cdot 7$ & $46 \cdot 8$ & 44. 9 & $47 \cdot 8$ & $52 \cdot 3$ & $50 \cdot 1$ & $54 \cdot 6$ & $61 \cdot 2$ & $59 \cdot 2$ & 63.7 & $70 \cdot 2$ & 68.0 & $74 \cdot 1$ \\
\hline Fat & 48.1 & 50.2 & $46 \cdot 1$ & 37.2 & 39.9 & 35.9 & $31 \cdot 1$ & 33.4 & $27 \cdot 1$ & 27.5 & 28.9 & $26 \cdot 1$ & $15 \cdot 4$ & 16.9 & $12 \cdot 2$ \\
\hline Protein & 10.5 & 11.2 & $9 \cdot 2$ & 9.7 & 9.5 & 9.9 & 9.5 & 9.7 & 9.4 & 9.4 & 9.5 & $9 \cdot 2$ & $8 \cdot 8$ & 9.2 & 8.3 \\
\hline $\mathrm{GL}$ & $163 \cdot 6$ & $159 \cdot 0$ & $178 \cdot 7$ & $221 \cdot 3$ & 211.0 & 231.4 & $253 \cdot 1$ & $248 \cdot 6$ & $259 \cdot 8$ & $312 \cdot 3$ & $301 \cdot 2$ & 323.6 & $367 \cdot 6$ & 354.9 & $377 \cdot 9$ \\
\hline \multicolumn{16}{|l|}{ Medical } \\
\hline Cereal intake $(\mathrm{g} / \mathrm{d})$ & 169 & 162 & 175 & 248 & 241 & 256 & 293 & 285 & 299 & 347 & 329 & 365 & 477 & 483 & 467 \\
\hline Fat intake $(\mathrm{g} / \mathrm{d})$ & 131.0 & $135 \cdot 0$ & $128 \cdot 0$ & 109.5 & 118.9 & $100 \cdot 8$ & 93.9 & $92 \cdot 6$ & $95 \cdot 7$ & 81.6 & $83 \cdot 8$ & $77 \cdot 8$ & $60 \cdot 1$ & $58 \cdot 1$ & 63.2 \\
\hline \multicolumn{16}{|c|}{ Energy contribution (\%) } \\
\hline Carbohydrates & 31.8 & $28 \cdot 7$ & 33.4 & $41 \cdot 1$ & $39 \cdot 2$ & 43.9 & $50 \cdot 8$ & $49 \cdot 2$ & 51.9 & 54.9 & 53.2 & $55 \cdot 8$ & $66 \cdot 4$ & $69 \cdot 6$ & 63.3 \\
\hline Fat & $45 \cdot 8$ & $47 \cdot 2$ & 43.6 & $40 \cdot 4$ & $42 \cdot 9$ & 37.7 & $38 \cdot 1$ & 36.5 & 40.4 & 32.9 & 34.9 & 30.2 & $20 \cdot 4$ & $17 \cdot 8$ & 23.5 \\
\hline Protein & $12 \cdot 3$ & $13 \cdot 6$ & $11 \cdot 3$ & $11 \cdot 8$ & $12 \cdot 0$ & 11.5 & 11.5 & $12 \cdot 2$ & 11.1 & 11.4 & 11.0 & 11.8 & $10 \cdot 7$ & 11.6 & $10 \cdot 3$ \\
\hline GL & $126 \cdot 5$ & $119 \cdot 4$ & 130.5 & 183.5 & 176.5 & $199 \cdot 8$ & $220 \cdot 7$ & 216.5 & $229 \cdot 8$ & $262 \cdot 89$ & $254 \cdot 3$ & 276.5 & $341 \cdot 2 \cdot 0$ & $351 \cdot 0$ & 338.7 \\
\hline \multicolumn{16}{|l|}{ Surgical } \\
\hline Cereal intake (g/d) & 249 & 245 & 254 & 299 & 276 & 321 & 367 & 342 & 388 & 421 & 416 & 431 & 489 & 501 & 465 \\
\hline Fat intake $(\mathrm{g} / \mathrm{d})$ & 109.5 & $119 \cdot 0$ & 102.7 & 92.5 & 95.3 & 87.9 & $92 \cdot 6$ & 94.2 & $90 \cdot 1$ & 81.6 & 83.4 & 78.8 & $59 \cdot 1$ & 57.3 & $62 \cdot 1$ \\
\hline \multicolumn{16}{|c|}{ Energy contribution (\%) } \\
\hline Carbohydrates & 43.6 & $40 \cdot 2$ & 45.9 & 49.5 & 47.4 & $51 \cdot 6$ & $55 \cdot 1$ & $52 \cdot 1$ & $59 \cdot 1$ & 63.2 & 59.6 & $67 \cdot 7$ & 72.9 & 74.9 & $67 \cdot 8$ \\
\hline Fat & $41 \cdot 1$ & 44.7 & 38.6 & 35.5 & 37.5 & $32 \cdot 3$ & 30.5 & $32 \cdot 0$ & 28.5 & $24 \cdot 1$ & $26 \cdot 7$ & $22 \cdot 0$ & $15 \cdot 4$ & 13.5 & $17 \cdot 6$ \\
\hline Protein & 10.5 & $10 \cdot 0$ & $11 \cdot 1$ & $10 \cdot 1$ & $10 \cdot 3$ & $9 \cdot 7$ & 9.9 & $10 \cdot 2$ & 9.8 & $9 \cdot 7$ & 9.5 & 9.9 & $9 \cdot 2$ & 8.9 & $9 \cdot 4$ \\
\hline $\mathrm{GL}$ & $195 \cdot 4$ & $187 \cdot 6$ & 199.9 & $219 \cdot 6$ & $217 \cdot 4$ & 223.6 & $280 \cdot 3$ & $267 \cdot 8$ & 293.4 & $319 \cdot 2$ & 303.7 & 334.5 & 377.5 & 389.9 & $356 \cdot 7$ \\
\hline
\end{tabular}


the dietary GL in the Chinese dietary pattern could further reflect the ratios of carbohydrate and fat intake with the same energy intake. It was demonstrated in the present study that adult patients hospitalised in internal medical departments had lower dietary GL per individual per $d$ than surgical patients, while male patients had higher dietary GL per individual per $\mathrm{d}$ than females. According to the dietary nutrient references for Chinese citizens published by the Chinese Society of Nutrition in 2002, the optimal percentage in total energy intake is $55 \%$ to about $65 \%$ for carbohydrates and $20 \%$ to about $30 \%$ for fats ${ }^{(15)}$. In patients from internal medical departments with dietary GL lower than the 75th percentile, energy ratios were $<55 \%$ for cereals and $>32 \%$ for fats (much higher than is recommended). Thus, dietary patterns in patients hospitalised in internal medical departments failed to follow the requirement of a rational diet (sufficient cereals and limited animal foods) while those from surgical departments had relatively rational dietary patterns. It should be mentioned that patients from internal medical departments whose dietary GL was higher than the 95th percentile had a higher prevalence of overweight, obesity and the metabolic syndrome. However, their intakes are close to the dietary nutrient references for Chinese citizens (published by the Chinese Society of Nutrition in 2002) ${ }^{(15)}$. Disease onset is presumably due to factors other than dietary pattern, for example, total daily energy intake, type of foods, as well as way of cooking.

We observed in the present study that patients with a low dietary GL in either internal medical or surgical departments, and that patients from internal medical departments with a high dietary GL had a higher prevalence of obesity, overweight and the metabolic syndrome. This is suggestive that either an excessively high or low dietary GL is not preferred. The overall prevalence of obesity, overweight and the metabolic syndrome was higher in patients from internal medical departments than in those from surgical departments. It is notable that significantly higher prevalences of overweight/ obesity and the metabolic syndrome were observed in male patients with dietary GL levels lower than the 5th percentile, and in female patients with dietary GL levels higher than the 95th percentile. This indicates that a sex discrepancy may exist in obesity-prone dietary patterns, though further investigations are required. In all the patients studied, the prevalence of overweight/obesity was the highest among all diseases. Obesity poses great threatens to the health of patients and has been identified as a pathological status and one of the fundamental causes of other metabolic diseases. It has been reported that higher prevalences of hypertension, CHD, diabetes, abnormal blood lipids, stroke, gout, arthritis, varicose veins of the lower limbs and certain kinds of cancer are observed in patients with even slight or moderate obesity. When BMI level exceeds $25 \mathrm{~kg} / \mathrm{m}^{2}$, fatality of patients shows a positive linear correlation with the increasing $\mathrm{BMI}^{(16)}$. Fat accumulation of internal organs in obese patients is a cardinal feature of the metabolic syndrome, and is also a major cause of insulin resistance. Mediators secreted by adipocytes are closely associated with metabolic diseases ${ }^{(17)}$. Patients hospitalised in internal medical departments are generally aged individuals who are often compromised with 
multiple diseases, poor systemic conditions and inadequate physical activity. An irrational diet may exacerbate their underlying obesity, abnormal blood glucose and lipids, and may prolong the duration of hospitalisation.

The evaluation of dietary GL is closely related to its specific food origin. Therefore, the application of dietary GL in guiding food selection in patients must take the specific disease and detailed food origin into consideration. The results in our present study are not conflicting with the recommended low-GI diet: GI reflects the quality of carbohydrates in a single food, which is independent of total carbohydrate intake. Dietary GL not only reflects the quality of dietary carbohydrates, it also is associated with the quantity of daily carbohydrates. Dietary GL and food GI are not related in a simple way, and a high dietary GL definitely does not equate to high-GI foods. Moreover, the specific food categories should be considered when low-GI foods are recommended. For example, whole cereals are recommended, as their GI is lower than that of refined cereals, while excessive animal foods including meats and animal oils are not recommended though their GI is much lower than that of whole cereals.

In conclusion, we observed that the dietary pattern in hospitalised adult Chinese reflected by dietary GL was irrational, and was associated with an elevated prevalence of metabolic diseases. The present study results may help nutritionists to evaluate the impact of foods on health, promote rehabilitation in patients, and shorten the duration of hospitalisation.

\section{Acknowledgements}

The present study received no funding.

All authors reviewed the contents of the manuscript, approved of its contents and validated the accuracy of the data. The three authors contributed equally to the study. L. L. designed the study and revised the manuscript; Y. Y. collected data of patients from internal medical departments and wrote the manuscript in English; L. W. collected data of patients from surgical departments, wrote the manuscript and was responsible for the statistical analysis of data.

There are no actual or potential conflicts of interest.

\section{References}

1. Salmerón J, Manson JE, Stampfer MJ, et al. (1997) Dietary fiber, glycemic load, and risk of non-insulin-dependent diabetes mellitus in women. JAMA 277, 472-477.
2. Brand Miller J, Colagiuri S \& Foster-Powell K (1997) The glycemic index is easy and works in practice. Diabetes Care 20, 1628-1629.

3. Weber C \& Schnell O (2009) The assessment of glycemic variability and its impact on diabetes-related complications: an overview. Diabetes Technol Ther 11, 623-633.

4. O'Reilly J, Wong J, Stephen HS, et al. (2010) Glycaemic index, glycaemic load and exercise performance. Sports Med 40, 27-39.

5. Yang G, Wang G \& Pan X (2002) China Food Composition 2002. Beijing: Peking University Medical Press.

6. Atkinson FS, Foster-Powell K \& Brand-Miller JC (2008) International tables of glycemic index and glycemic load values: 2008. Diabetes Care 31, 2281-2283.

7. Chen CM (2008) Overview of obesity in mainland China. Obes Rev 9, 14-21.

8. Wang Y, Mi J, Shan XY, et al. (2007) Is China facing an obesity epidemic and the consequences? The trends in obesity and chronic disease in China. Int J Obes (Lond) 31, 177-188.

9. Anonymous (2004) Suggestions about metabolic syndrome from Chinese Diabetes Society (article in Chinese). Chin J Diabetes 12, 156-161.

10. Liu S, Willet WC, Stampfer MJ, et al. (2000) A prospective study of dietary glycemic load, carbohydrate intake, and risk of coronary heart disease in US women. Am J Clin Nutr 71, 1455-1461.

11. Sahyoun NR, Anderson AL, Tylavsky FA, et al. (2008) Dietary glycemic index and glycemic load and the risk of type 2 diabetes in older adults. Am J Clin Nutr 87, $126-131$.

12. Augustin LS, Dal Maso DL, La Vecchia C, et al. (2001) Dietary glycemic index and glycemic load, and breast cancer risk: a case-control study. Ann Oncol 12, 1533-1538.

13. Ford ES \& Liu S (2001) Glycemic index and serum highdensity lipoprotein cholesterol concentration among US adults. Arch Intern Med 161, 572-576.

14. Franceschi S, Dal Maso L, Augustin L, et al. (2001) Dietary glycemic load and colorectal cancer risk. Ann Oncol 12, 173-178.

15. Yang G, Wang G \& Pan X (2002) China Food Composition 2002. Addition 2, Chinese Dietary Recommendation Intakes. Beijing: Peking University Medical Press.

16. Anderson PJ, Critchley JA, Chan JC, et al. (2001) Factors analysis of the metabolic syndrome: obesity $v s$ insulin resistance as the central abnormality. Int J Obes Relat Metab Disord 25, 1782-1788.

17. Grundy SM, Hansen B, Smith SC Jr, et al. (2004) Clinical management of metabolic syndrome: report of the American Heart Association/National Heart, Lung, and Blood Institute/American Diabetes Association conference on scientific issues related to management. Circulation 109, $551-556$. 